\title{
Israels Haltung zur Palästina-Frage - Konzepte und Hintergründe
}

\author{
Von Peter Gottstein
}

Seit dem Ausbruch des Aufstandes der palästinensischen Bevölkerung in den von Israel besetzten Gebieten, kurz: Intifada, im Dezember 1987 ist die nahöstliche Szene in Bewegung geraten. Von neuem Selbstbewußtsein getragen hat die Palästinensische Befreiungsorganisation (PLO) politische Schritte getan, die man ihr früher nicht zugetraut hätte: in einer mit großer Mehrheit gefaßten Resolution ihrer Legislative akzeptierte sie Israels Existenz und verschrieb sich ersmals einer Zwei-Staaten-Lösung für Palästina. Die Vereinigten Staaten honorierten dies mit der Aufnahme eines Dialogs mit der PLO.

Von den Entwicklungen offenkundig überrascht, fand die israelische Regierung der Nationalen Einheit (RNE) - im wesentlichen eine große Koalition aus dem nationalliberalen Likud und der Arbeiterpartei (AP) - zu keiner überzeugenden Antwort. Lange beschränkte sie sich auf Demarchen bei den westlichen Regierungen, die vom Verkehr mit der PLO abgehalten werden sollten und versuchte gleichzeitig, die Intifada mit polizeilichen Mitteln zu unterdrücken und die PLO militärisch zu schwächen.

Der flagrante Mißerfolg dieser Politik und die daraus resultierenden gravierenden Probleme für Israels Wirtschaft, Gesellschaft und Außenbeziehungen haben innenpolitische Prozesse in Gang gesetzt, deren Ergebnis kaum vorhersehbar scheint. Von der öffentlichen Meinung und der Entwicklung der Machtverhältnisse hängt jedoch ab, welche Haltung Israel in der Frage einer Friedenslösung im allgemeinen und gegenüber der PLO im speziellen einnimmt. Den sehr entgegenkommenden Vorstellungen auf dem linken Flügel des israelischen Parteienspektrums stehen unnachgiebige und extremistische Konzepte auf dem rechten gegenüber. Nicht zuletzt wegen des seit einigen Jahren bestehenden politischen Patts zwischen Likud und Arbeiterpartei haben die kleinen Parteien, die sich solche Positionen zu eigen gemacht haben, an Zulauf gewonnen. Die große Publizität für ihre Aktionen - seien dies auf der einen Seite Treffen mit PLO-Funktionären oder Ausschreitungen gegen Palästinenser und illegale Neugründungen von Siedlungen in den Territorien auf der anderen - täuscht aber darüber hinweg, daß die beiden großen Parteien über eine sehr komfortable absolute Mehrheit sowohl im israelischen Parlament als auch in den Kommunen verfügen. Unter den gegebenen Umständen kann also davon ausgegangen werden, daß zumindest die Vorstellungen einer der beiden Parteien, wenn nicht beider gemeinsam in absehbarer Zeit ausschlaggebend für die Formulierung der israelischen Palästina-Politik sein werden. 
Zur Beurteilung der weiteren Entwicklung in der Region erscheint es daher aufschlußreich, die Konzepte von Likud und Arbeiterpartei, wie sie bis zum Wahlkampf 1988 in relativ umfassender Weise entwickelt wurden, den Grundpositionen der PLO gegenüberzustellen.

\section{Mehrheitsfähige Friedenskonzepte in Israel und das politische Programm der PLO - eine Gegenüberstellung}

\section{Die Modalitäten des Friedensprozesses}

\section{a. Likud}

Die Vorstellungen des Likud über die Modalitäten eines politischen Friedensprozesses bauten schier unverrückbar auf dem Vertragswerk von Camp David auf, in dem vor allem der Eintritt in direkte Verhandlungen mit Jordanien und allen anderen arabischen staatlichen Konfliktparteien vorgesehen ist.

Eine internationale Friedenskonferenz wurde grundsätzlich abgelehnt. Dies galt gleichermaßen für alle diskutierten Varianten. Für den Likud kam weder ein rein zeremonieller "internationaler Rahmen" noch ein internationales Beschlußgremium in Frage. Für den Fall direkter Verhandlungen mit Jordanien bot man allerdings an, diese unter ägyptischen Auspizien abzuhalten. Von einer Beteiligung des "palästinensischen Volkes" war keine Rede, wohl aber von einer "autoritativen und glaubwürdigen, jedoch nicht gewählten" Repräsentation der palästinensischen Bevölkerung Judäas, Samarias und Gazas. Diese Verteter dürften keine Bindungen an die PLO haben, müßten also wohl im Rahmen der jordanischen Delegation auftreten.1 Von der US-Regierung zu Zugeständnissen gedrängt, hat Premierminister Schamir seine Ablehnung gegenüber Wahlen relativiert. Allerdings haben die von ihm gestellten Bedingungen - israelische Aufsicht nach den Wahlen, vorherige Beendigung der Intifada, keine PLO-Beteiligung - auf der arabischen Seite keine Zustimmung erhalten.2

b. $A P$

Auf im wesentlichen bilateral geführte Verhandlungen setzte auch die AP. Seit 1986 gesteht sie in Anerkennung politischer Bedürfnisse speziell Jordaniens zu, daß diese in

1 Vgl. das Interview in: Summary of World Broadcasts/Middle East (SWB/ME), 13.9.1988, S. 5; vgl. Schamir, Jitzhak, Israel at 40, in: Foreign Affairs, Vol.66, No.3 (1988), S. 578; Interviews, in: Intemational Herald Tribune (IHT), 25.4.1987, S. 2, und Le Monde (LM), 26.2.1985, S. 3.

2 Vgl. Neue Zürcher Zeitung (NZZ), 2.4.1989, S. 2, SZ 17.4.89, S. 8. 
einen zeremoniellen internationalen Rahmen gebettet werden. Das internationale Element sollten dabei die permanenten Mitgliedsstaaten des VN-Sicherheitsrates, USA, Sowjetunion, Frankreich, Großbritannien und China liefern. Anfangs hatte Peres allerdings die Beteiligung Chinas und der UdSSR durch eine Klausel in Frage gestellt. Die Bedingung, daß alle dafür vorgesehenen (nicht-arabischen) Teilnehmerstaaten a priori normale Beziehungen mit Israel haben müßten, wurde aber inzwischen wohl fallengelassen. 3

Die palästinensische Bevölkerung in den besetzten Gebieten sollte nach dem Konzept der AP - entweder in einer eigenen oder einer gemeinsamen Delegation mit Jordanien vertreten sein. Deren Mitglieder müßten "Terror und Gewalt" abgeschworen und die Existenzberechtigung Israels anerkannt haben. Auch dürften sie keine förmlichen Mitglieder der PLO sein. Zwar sollte die Biographie der Teilnehmer nicht allzu genau durchleuchtet werden, doch verdeutlicht dieser Passus, daß Israel offensichtlich eine Begutachtung der Teilnehmer vorbehalten bleiben sollte. 4 Als Reaktion auf die Verzichtserklärung des jordanischen Monarchen vom Sommer 1988 wurde außerdem in letzter Minute die Vorstellung entwickelt, im Zweifelsfall nicht auf Verhandlungen mit einer vereinten jordanisch-palästinensischen Delegation zu bestehen. Vielmehr sollten nunmehr auch separate jordanische und palästinensische Delegationen akzeptiert, die Vertreter der letzteren in freien Wahlen von der Bevölkerung der besetzten Gebiete bestimmt werden. 5 Die Freiheit der Wahlen wurde allerdings dadurch eingeschränkt, daß die PLO von einer Kandidatur ausgeschlossen sein sollte. 6 Der im Januar 1989 von Verteidigungsminister Rabin vorgelegte Plan deckt sich mit diesen Vorstellungen weitgehend. Er führt allerdings die neue Bedingung ein, daß zunächst in einer Phase von drei bis sechs Monaten die "Ordnung" in den Gebieten wiederhergestellt, d.h. die Intifada beendet werden müsse.7

\section{c. $P L O$}

Die Vorstellungen der RNE standen im Widerspruch zu den Positionen der PLO. Sie negierten das Faktum, daß die PLO das Recht auf alleinige und souveräne Vertretung des palästinensischen Volkes beansprucht und jegliche Differenzierung zwischen Palästinensem "innerhalb und außerhalb der besetzten Gebiete" strikt ablehnt. 8 In beidem wird die

3 Vgl. die klare Ablehnung einer Beteiligung der UdSSR an einer Nahost-Friedenslösung in seinem Interview in: Politique Intemationale, No. 28 (Sommer 1985), S. 17. In den späteren Äußerungen fehlt dieser Punkt.

4 Vgl. das Interview mit Peres in: IHT, 26.9.1988, S. 5.

5 Vgl. Jerusalem Post, 29.9.1988, S. 5; NZZ, 20.10.1988, S. 2.

6 ebd.

7 Vgl. SWB/ME vom 21.1.89, S. 2.

8 Vgl. die Erklärung des PLO-Exekutivkomitees, Tunis, 7.3.1983, zit. nach: Joumal of Palestine Studies (JPS), Vol. 15, No. 4/60 (Sommer 1986), S. 234. 
PLO von einer überwältigenden Mehrheit der Palästinenser getragen. Freilich läßt sich das mangels eines Rechts auf freie Meinungsäußerung zu dieser Frage und mangels freier Wahlen für die Palästinenser in allen ihren nahöstlichen Gastländern nur informell ermitteln. Die derzeit umfangreichste Meinungsumfrage, 1986 von der Ost jerusalemer Zeitung "al-Fajr" durchgeführt, darf allerdings als deutlicher Hinweis zur Sache gewertet werden. Notabene hat die Umfrage auch eine große persönliche Mehrheit für den Vorsitzenden der Organisation "al-Fatah" und PLO-Chef, Jassir Arafat, ergeben. Dieser Befund konnte auch durch Gespräche mit Palästinensem im In- und Ausland bestätigt werden.9

Hinter der Ablehnung direkter und bilateraler Verhandlungen mit Israel sowohl für sich als auch für die anderen arabischen Staaten stand und steht vor allem die Sorge der PLO vor einer Situation, in der Israel dank seiner militärischen Überlegenheit und dank der als kompromißlos empfundenen Unterstützung durch die USA einen Frieden diktieren könnte.10

Das Bestehen auf Entscheidungsvollmacht der befürworteten gewünschten internationalen Konferenz, an der neben allen arabischen Staaten auch die fünf ständigen Mitglieder des VN-Sicherheitsrates teilnehmen sollten, spielte daher eine wichtige Rolle. Sie sollte verhindern helfen, daß die Interessen der arabischen Staaten undurchsetzbar würden, wenn erst einmal deren einziger Trumpf aus der Hand gegeben wäre: Dies wäre dann der Fall, wenn Israel beschlösse, daß es mit dem Faktum von Verhandlungen seine Hauptinteressen Überwindung der politischen Isolation und damit verbunden, faktische Anerkennung seiner Legitimität - verwirklicht hätte. Es ist allerdings möglich, daß die PLO dank der ihr gewährten ägyptischen und jordanischen Unterstützung zu größerer Flexibilität in diesem Punkt findet.11

9 Vgl. etwa die Meinungsumf rage der Ost-Jerusalemer Zeitung al-Fajr, veröffentlicht in Washington am 8.9.1986 als al-Fa jr Public Opinion Survey; zit. nach JPS, Vol. 16, No. 2/62 (Winter 1986/87), S. 196-207. Solche Gespräche konnte der Verfasser seit 1984 u.a. mit in Syrien und in Israel lebenden Palästinensem führen.

10 Vgl. Interview mit Arafat in: The Middle East, No. 149 (März 1988), S. 21. Die Perzeption, daß die USA Israel blindlings unterstützen, wird wohl von allen arabischen Ländern geteilt. Vgl. Nahmes, Victor, Israel in Jordan's eyes, in: Jerusalem Post, 8.1.1986. Dies gilt in ganz besonderem $\mathrm{Maße}$ auch für Syrien. Die in der Außenpolitik des Landes relevanten Perzeptionen werden gegenwärtig vom Verf. in einer Studie über "Leitlinien und Hintergründe syrischer Außenpolitik" untersucht.

11 Vgl. NZZ, 29.3.1989, S. 3. 


\section{Inhalte einer politischen Lösung}

\section{a. Likud}

Israelische Kontrolle über die besetzten Gebiete war Dreh- und Angelpunkt der Lösungsvorschläge des Likud. Angesichts der Brisanz des Themas ist nicht verwunderlich, daß Schamir als Parteivorsitzender und Premierminister dazu keine Details bekanntgab. Nach dem jordanischen Rückzug aus administrativer Verantwortung für das West jordanland, von König Hussein am 31.Juli 1988 erklärt, sprach sich Schmamir jedoch in unerwarteter Offenheit gegen Annexionspläne aus, die in seiner Partei zirkulierten.12 Er berief sich dabei allerdings auf die offizielle Politik des Likud-Blocks und auf das Wahlprogramm, nicht aber auf die grundsätzliche Ideologie der Partei. So blieb ein Widerspruch bestehen, da die Gewinnung des ganzen "Eretz Israel" für das jüdische Volk von Anfang an zu den festen Glaubenssätzen der Partei, wie sie von Menachem Begin geprägt wurde, gehörte. 13 Möglicherweise haben aber Elemente der Liberalen Partei, deren endgültige Verschmelzung mit Schamirs Cherut-Partei im August 1988 aus dem Likud-Block die Likud-Partei machte,14 eine Rolle bei der Abwertung der groß-israelischen Ideologie zugunsten des relativ verhalteneren Wahlprogramms gespielt. Als sicher kann jedoch gelten, daß Schamir und die Partei an dem Recht der israelischen Bürger, in allen Gebieten zu siedeln, festhielten.15 Inwieweit damit noch ein unmittelbares, die Tagespolitik prägendes Interesse an territorialer Expansion verbunden war, läßt sich schwer beurteilen. Die Tatsache, daß sich die konsequenten Vertreter einer Annexion des Westjordanlandes und des Gaza-Streifens außerhalb des Likud etwa in der Techija-Partei und in noch kleineren one-issue-Parteien, sammelten, spricht jedenfalls gegen eine solche Annahme. Wichtiger aber ist, daß eine Annexion den Friedensvertrag mit Ägypten - erklärte Grundlage gerade der Außenpolitik Premierminister Schamirs - gegenstandslos machen dürfte. 16

Den Bestrebungen der palästinensischen Bevölkerung sollte durch Autonomie Rechnung getragen werden. Nach einer Frist von fünf Jahren sollte dann mit der palästinensischen Führung der Gebiete über die Frage der Souveränität verhandelt werden. Die grundsätzliche Position des Likud lautete allerdings, daß ein souveräner Palästinenser-Staat weder wirtschaftlich noch unter dem Aspekt israelischer Sicherheitsinteressen in Frage käme. Letztere sah Schamir auch im Falle einer Angliederung der Gebiete an Jordanien so gravierend bedroht, daß auch diese Option für ihn ausgeschlossen blieb.17 So blieb unklar, was nach

12 Vgl. z.B. die Äußerungen Minister Ariel Scharons, in: Jerusalem Post, 17.8.1988, S. 12.

13 Zur Ideologie des Likud vgl. Orland, Nachum, Die Cherut. Analyse einer rechtsorientierten israelischen Partei, München 1983.

14 Vgl. Jerusalem Post, 26.8.1988, S. 20.

15 Vgl. Schamir, Israel at 40, S. 579.

16 Vgl. ebd., S. 579, und seine Rede vom 10.8.1988.

17 Vgl. Süddeutsche Zeitung (SZ), 19.10.1988, S. 7; Schamir, Israel at 40, S. 576. 
Ablauf der fünfjährigen Autonomie-Periode Gegenstand der in Aussicht gestellten Souveränitätsverhandlungen sein sollte.

\section{b. $A P$}

Im Konzept der AP wurde diese Frage beantwortet. Zunächst baute allerdings auch sie auf eine befristete Autonomie als ersten Schritt einer Ubereinkunft. Begrenzt auf einen Zeitraum von fünf Jahren sollten der palästinensischen Selbstverwaltung nur im Bereich der Sicherheit Einschränkungen auferlegt werden. Im Interesse israelischer Bedürfnisse sollten die Territorien entmilitarisiert werden. Dies würde im Gebiet westlich des Jordans nicht nur für etwaige palästinensische Verbände gelten, sondern auch für die jordanische oder jede andere arabische Armee. Von entscheidender Bedeutung gerade im Vergleich zu dem Konzept des Likud ist, daß die nicht-militärische Kontrolle über das West jordanland an Jordanien abgetreten werden sollte, mit Ausnahme von Gebieten lebenswichtiger strategischer Bedeutung und der Stadt Jerusalem, deren erneute Teilung die AP ebenso kategorisch ablehnte wie der Likud. Der Gaza-Streifen sollte dagegen vollständig geräumt werden.18 Anzumerken ist, daß die AP trotz der Erklärung König Husseins an ihrem Konzept einer Hauptrolle Jordaniens festhielt. Offensichtlich lag dem die Annahme zugrunde, daß dieser Schritt durchaus umkehrbar wäre und daß das Königreich für eine Föderation mit den Gebieten gewonnen könne. Ein unabhängiger Palästinenser-Staat zwischen Israel und Jordanien war in der Sicht der AP nicht weniger ausgeschlossen als für den Likud.19

\section{c. $P L O$}

Mit der Likud-Vorstellung, daß palästinensische Selbstbestimmung auf eine Art von Kommunalpolitik unter israelischer Aufsicht beschränkt bleiben sollte, war die PLO natürlich ganz und gar nicht einverstanden. Dies gilt unabhängig von der Tatsache, daß darin grundsätzlich keine Rolle für die PLO vorgesehen war.

Aber auch das Konzept der AP war nicht akzeptabel für die PLO, die weder bereit war, sich jordanischer Herrschaft zu beugen, noch die von der AP vorgeschlagenen Wahlen unter der Bevölkerung der besetzten Gebiete anzunehmen.20 Daß bei den Wahlen keine Vertretung der in arabischen Ländern lebenden Flüchtlinge vorgesehen war, wurde als Versuch Israels

18 Vgl. das Wahlprogramm der AP in: SWB/ME, 26.4.1988; Erklärung Peres'in Paris, in: LM, 25.9.1988, S. 3.

19 Vgl. Wahlprogramm der AP, a.a.O.; Interview mit Verteidigungsminister Jitzhak Rabin, nach JPS, Vol. 17, No. 3, S. 156. Auch Rabin hält in seinem Plan in großer Deutlichkeit daran fest, daß die Sicherheit in den Gebieten in der Kompetenz der israelischen Streitkräfte bleiben müsse.

20 Vgl. die prompte Ablehnung des AP-Plans durch die PLO, in: Jerusalem Post, 19.10.1988, S. 1. 
gewertet, einen Keil zwischen Flüchtlinge und PLO auf der einen und den Dagebliebenen samt ihrer Führung auf der anderen Seite zu treiben. Die PLO forderte dagegen die Errichtung eines unabhängigen Staates mit allen Hoheitsrechten. Erst danach bestünde die Möglichkeit, daß sich dieser mit Jordanien zu einer Konföderation gleichberechtigter Partner zusammenschlösse. 21

Die von der AP geforderte Entmilitarisierung der Territorien ist zwar verschiedentlich von einzelnen Persönlichkeiten aus den besetzten Gebieten akzeptiert worden, 22 doch in offiziellen Dokumenten und Äußerungen der PLO findet sich derlei nicht: Israelischen Sicherheitsbedürfnissen könnte im militärischen Bereich durch die vorübergehende Stationierung von VN-Truppen und im politischen Bereich durch Verträge nachgekommen werden. Ein Verzicht auf sonstige Hoheitsrechte kam jedoch für die PLO offensichtlich nicht in Frage.23

Israelische Souveränität über die gesamte Stadt Jerusalem wurde abgelehnt, denn zumindest deren arabischer Teil soll ja Hauptstadt des palästinensischen Staates werden. Abgelehnt wurden auch die israelischen Siedlungen auf dem Territorum des zu gründenden Staates, zumindest solange diese nicht unter palästinensisches Recht gestellt würden.24 Eine Absicht, die besetzten Gebiete "judenrein" zu machen - wie Jitzhak Schamir befürchtet 25 - konnte in keinem Fall nachgewiesen werden.

Die in den Konzepten von Likud und Arbeiterpartei ausgedrückte strikte Ablehnung der PLO und ihrer politischen Positionen ist erklärungsbedürftig, denn schließlich ist Israel bereit, mit seinen ärgsten arabischen Gegnern, den syrischen Präsidenten eingeschlossen, zu verhandeln. 26 Die Vermutung liegt nahe, daß die PLO für Israel eine Art politisches

21 Vgl. Drei Vorschläge der PLO zur Klärung ihrer Position zum Friedensprozeß, amerikanischen und jordanischen Unterhändlem vorgelegt in Amman am 5.2.1986, in: JPS, Vol. 15 No 4/60 (Sommer 1986), S. 241f.

22 Vgl. Siniora, Hana, Deux nations dans le même pays, in: LM, 1.4.1988, S. 6

23 Vgl. Arafats Rede vor der sozialistischen Gruppe im Europa-Parlament, in: SWB/ME, 16.9.1988, S. 4. Die altemative Idee, statt der VN-evtl. auch "europäische Truppen" zu stationieren, entsprang wohl dem Genus loci Straßburgs und braucht nicht weiter berücksichtigt zu werden; vgl. die Erklärung des Palästinensischen Nationalrats, Algier, 27.4.1987, nach JPS, No. 64 (Sommer 1987), S. 203f.

24 Vgl. Arafat-Rede in: SWB/ME, 16.9.1988, S. 4 sowie die Erklärung des Palästinensischen Nationalrats, Algier, 27.4.1987, nach JPS, Vol. 16 No 4/64 (Sommer 1987), S. 203f. Arafat und andere haben immer wieder betont, daß es in einem palästinensischen Staat keine religiöse oder sonstige Diskniminierung geben werde. Die Tatsache, daß bis dato innerhalb der PLO keine offenen religiös oder konfessionell geprägten Konflike zu verzeichnen gewesen sind, verleiht dem Glaubwürdigkeit.

$25 \mathrm{Vgl}$. Schamir, Israel at 40, S. 579.

26 Vgl. z.B. die Erklärung Schamirs vor der Knesset am 10.8.1988; Memograph, dem Verfasser zur Verfügung gestellt von der israelischen Botschaft in Bonn. 
Allergen darstellt. Im folgenden gehen wir daher der Frage nach, welche grundlegenden Werte, Sichtweisen und Interessen des Staates Israel und seiner Bevölkerung durch die politischen Ziele und den bewaffneten Kampf der PLO in Frage gestellt werden.

\section{Zum Hintergrund israelischer Palästina-Politik}

\section{Zur Frage der Legitimität des Staates Israels}

Die Frage nach der Legitimität Israels spielt noch heute in der politischen und propagandistischen Auseinandersetzung im Nahen Osten eine wichtige Rolle. Sie berührt völkerrechtliche Aspekte, aber insbesondere auch umfassende normative Konzepte, die der Erläuterung bedürfen. Im Interesse der analytischen Schärfe wird hiervon die raison d'être Israels unterschieden, die im folgenden als die Summe der selbstgewählten Staatsaufgaben aufgefaßt wird.

Nach den Ausführungen eines namhaften deutschen Israel-Kenners ist davon auszugehen, $\mathrm{da} ß$ die israelische Staatsgründung nicht wegen, sondern trotz des Holocausts zustandegekommen ist.27 Seiner Argumentation, daß die Unterstützung und Zustimmung der Vereinten Nationen, der USA oder der Sowjetunion für den Erfolg des jüdischen Nationalismus belanglos gewesen sei, muß man so nicht folgen.28 Doch läßt sich in der Tat feststellen, $\mathrm{da} \beta$ in den Augen der Zionisten zumindest die Legitimität der Staatsgründung ganz unabhängig vom Holocaust gegeben war. Zu den Quellen dieser Legitimität zählt der Teilungsbeschluß der Vereinten Nationen vom 29. November 1947 erst an nachgeordneter Stelle.

Wichtiger als das Völkerrecht war den Staatsgründern unter Führung David Ben-Gurions das natürliche und historische Recht, kraft dessen sie zu handeln glaubten.29 Diese Sicht ist nach wie vor von grundlegender Bedeutung für die israelische Politik. Das historische Recht der Juden auf Judäa, Samaria und sogar Galiläa - dem heutigen Territorium Israels einschließlich der besetzten Gebiete sowie Teilen des Südlibanons - wurde mit der Anciennität israelischer Staatlichkeit in der Region begründet. So argumentierte etwa Jitzhak Schamir, daß die "jüdische die einzige unabhängige nationale Souveränität sei, die in der dreitausend Jahre alten Geschichte der Region...existiert" habe. 30 Für einen palästinensischen Nationalismus, wie ihn etwa die PLO vertrete, fehle dagegen jegliche Grundlage.

27 Vgl. Wolffsohn, Michael, Ohne Hitler kein Israel? Eine historische Einordnung, in: SZ, 21.4.1988, S. 11 .

28 Vgl. Meir, Golda, Mein Leben, Hamburg 1975, S.212f und S. 233f. Die schnelle Anerkennung des jungen Staates durch die beiden Großmächte war zumindest in den Augen der späteren Premieministerin "von ungeheurer Bedeutung".

29 Vgl. Schreiber, Friedrich/Wolffsohn, Michael, Nahost. Geschichte und Struktur des Konflikts, Opladen 1988, S. 128.

30 Schamir, Jitzhak, Israel at 40, in : FA, Vol. 66, No. 3 (1988), S. 576. 
Noch in der Zwischenkriegszeit hätten sich die arabischen Bewohner des Landes nämlich dagegen gewehrt, als Palästinenser bezeichnet zu werden, weil sie sich der großen arabischen Nation zugehörig gefühlt hätten. Vom "historischen Recht des Volkes von Israel auf sein Land" spricht man aber auch in der AP mit großer Selbstverständlichkeit.31

Im Unterschied zu der Selbstverständlichkeit, mit der die Deutschen und andere Völker auf ihre jeweilige staatliche Existenz schauen, besteht die Besonderheit des in Israel vorherrschenden staatlichen Selbstverständnisses also darin, daß die Legitimität der Existenz Israels im wesentlichen nicht aus der normativen Macht des Faktischen abgeleitet wird. Daher besitzen die Ansiedlung jüdischer Einwanderer in Palästina, die Gründung des Staates Israel und dessen Verteidigung aus israelischer Sicht eine "höhere Legitimität" als die Ansprüche der arabischen Bevölkerung.

\section{Zur raison d'être des jüdischen Staates}

Die raison d'être des Staates Israel steht sehr wohl in engem Zusammenhang mit der Katastrophe des jüdischen Volkes. Die israelische Unabhängigkeitserklärung drückt dies wohl am prägnantesten aus. Die Staatsziele "ungehinderte jüdische Einwanderung" und "Sammlung des zerstreuten Volkes" sind zwar auch ohne den Holocaust plausibel. Ihre dramatische Bedeutung erhielten sie aber doch erst vor dem Hintergnund der unendlichen Schwierigkeiten und Frustrationen, auf die jüdische Hilfsorganisationen bei ihrem Bemühen stießen, möglichst vielen Juden die Flucht nach Palästina sowie in andere Länder zu ermöglichen.32 Es war daher kein Zufall, daß die erste Amtshandlung der provisorischen Regierung darin bestand, die von den britischen Mandatsbehörden erlassene Einwanderungsbeschränkung aufzuheben. 33

Ein weiteres wesentliches Element des staatlichen Selbstverständnisses war Wehrhaftigkeit. Diese Eigenschaft erwies sich bereits in der teilweise sehr blutigen Auseinandersetzung der jüdischen Siedler mit der arabischen Bevölkerung Palästinas vor allem in den späten 20er und durch die 30er Jahre hindurch als notwendig. 34 Prägend wurde aber auch hier der Holocaust mit seinen Berichten und Bildern von den Millionen, die hilf- und wehrlos ihrer Vernichtung entgegengingen. Derlei sollte sich nie wieder abspielen können. Nie wieder sollte das jüdische Volk seinen Feinden wehrlos ausgeliefert sein. 35

31 Vgl. das Interview des Verteidigungsministers Jitzhak Rabin, in: Jane's Defence Weekly (JDW), 17.3.1988, S. 156.

32 Vgl. etwa Meir, Mein Leben, S. 182.

33 Vgl. Archiv der Gegenwart (AdG), 21.4.1948, S. 1498; Meir, Mein Leben, S. 229 und 232.

34 Vgl. Bethell, Nicholas, The Palestine Triangle, London 1979, S. 36.

35 Die historische Lehre aus dem Holocaust spielte bei der Beschlußfassung so unterschiedlicher Persönlichkeiten wie Golda Meir und Menahem Begin gleichermaßen eine große Rolle. Zur Entschei- 


\section{Die Perzeption arabischer Bedrohung im Spiegel der Holocaust-Erfahrung}

Zu den folgenschwersten Entwicklungen der Nachkriegszeit in der Region dürfte gehören, $\mathrm{da}$ die Erfahrung der unbeschreiblichen Massenvemichtungsmaschinerie der Nationalsozialisten in israelischer Wahmehmung mit der Bedrohung durch die feindselige arabische Bevölkerung und besonders mit der militärischen Aggression der arabischen Staaten zu einer neuen Bedrohungsperzeption verschmolz.36 Die Erklärungen der arabischen Regierungen 1947/48, keine jüdische Staatsgründung zulassen zu wollen und eine solche als Casus belli aufzufassen, 37 wurde unter dem Aspekt existentieller Bedrohung interpretiert. Dies galt genauso für spätere arabische Gelübde, den israelischen Staat zu zerschlagen. 38

1948 konnten an dieser Sicht auch die lange geheimgehaltenen Kontakte der zionistischen Führung mit König Abdullah von Jordanien nicths ändern. Dieser hatte den jüdischen Siedlern den Verzicht auf einen eigenen Staat nahegelegt und dafür die Aufnahme der Juden als gleichberechtigte Untertanen in sein Reich angeboten. Natürlich war das Angebot für Ben-Gurion und seine Leute aus mehr als einem Grund nicht akzeptabel.39

Vor allem waren die Zionisten nach den noch allgegenwärtigen Erfahrungen damals nicht mehr bereit, ihre Sicherheit einer fremden Macht anzuvertrauen. Eine weitere Uberlegung von erheblicher Bedeutung trat hier aber noch hinzu: Im Königreich Transjordanien konnte es niemals eine jüdisch geprägte Gesellschaft geben. Nach Überzeugung der Zionisten mußte es aber einen Staat geben, in dem die Juden nicht nur bestenfalls geduldet, sondern endlich beheimatet, ja in der Mehrheit wären. 40

Das Interesse an einer "grundlegend jüdische(n) Natur des Staates Israel" hatte ja 1947 die große Mehrheit der zionistischen Führer zur Annahme des VN-Teilungsbeschlusses bewegt. Bis zum Sechs-Tage-Krieg war die Prämisse, daß ein jüdischer Staat in einem Teil von Palästina besser sei als ein binationaler im ganzen Land, de facto von allen Parteien

dung über den Präventivangriff gegen Ägypten 1967 vgl. Meir, Mein Leben, S. 306; zu Begins Argumentation für den Luftangriff auf den irakischen Atomreaktor 1981 vgl. Perlmutter, Amos, The Life and Times of Menahem Begin, Garden City, N.Y. 1987, S. 362.

36 Besonders greifbar wird dies in den Äußerungen israelischer Militärs nach dem Sechs-Tage-Krieg. Viele berichteten, sie hätten das Gefühl gehabt, in den arabischen Soldaten den "anonyme(n) Feind" des jüdischen Volkes zu bekämpfen und dabei den gegen die Nazis unterlassenen Widerstand nachzuholen; vgl. Neustadt, Amonon, Israels zweite Generation. Auschwitz als Vermächtnis, Berlin 1987, S. 30.

37 Vgl. Ben-Gurion, David, Wir und die Nachbam, Tübingen 1968, S. 427.

38 Vgl. Meir, Mein Leben, S. 306; Seligmann, Rafael, Israels Sicherheitspolitik. Zwischen Selbstbehauptung und Präventivschlag. Eine Fallstudie über Grundlagen und Motive, München 1982, s. 13f.

39 Vgl. Meir, Mein Leben, S. 217ff.

40 ebd., S. 288. 
akzeptiert. ${ }^{41}$ Die programmatische Opposition der Cherut-Partei unter Menachem Begin gegen diese Wertung wurde erst später wirksam. Unter seinem Nachfolger Schamir traten solche sozio-kulturellen Bedürfnisse ebenfalls zurück. Die konsequente Verwirklichung des auch von den übrigen Zionisten so verstandenen jüdischen Rechts auf Besiedelung des gesamten Eretz Israel und die Gewährleistung von Sicherheit, die vor allem in territorialen und militärischen Kategorien begriffen wird, sind bis heute die Leitsätze Jitzhak Schamirs und seines Likud-Blocks. 42

\section{Zur Bedeutung israelischer Staatsziele für die Politik der RNE}

Welche Rolle spielte das Selbstverständnis von der Legitimität und der raison d'être des Staates Israel für die Politik der RNE?

Die nach wie vor hohe Prioriät der Einwanderung von Juden ins Gelobte Land in der israelischen Politik wird schnell deutlich, wenn man die Bemühungen um Juden aller Nationalitäten in den Jahren 1984-1988 betrachtet. Markante Beispiele waren etwa die spektakuläre Umsiedlung einiger tausend äthiopischer Nomaden,43 das fortdauemde Engagement für die jüdische Gemeinschaft in der Sowjetunion oder die Bemühungen um die Auswanderung der noch in arabischen Ländem verbliebenen Juden nach Israel.

Was die Perzeption existenzieller Bedrohung angeht, so mag es dem Außenstehenden unwahrscheinlich vorkommen, daß derlei nach den wiederholten militärischen Erfolgen Israels gegen die arabischen Streitkräfte, dem Frieden mit Ägypten und dem strategischen Bündnis mit den USA von 1982 fortbestehen könnte. Jedoch wird auch in neueren IsraelDarstellungen darauf hingewiesen. Eine Untersuchung der israelischen Entscheidungsfindung in den Krisensituationen von 1967 und 1973 fördert z.B. zutage, daß 1967 unter den damaligen Entscheidungsträgem das sogenannte "Holocaust-Syndrom", d.h. konlerete Ängste vor einer Wiederholung des Unaussprechlichen, wirksam waren und artikuliert wurden.

Daneben war man entsprechend Ben-Gurions Dogma krampfhaft um die Unterstützung der Großmächte bemüht und lebte in dem ebenfalls typischen Gefühl der Isolation und der

41 Vgl. Eisenstadt, Shmuel N., Die Transfornation der israelischen Gesellschaft, Frankfurt 1987, S. 247.

42 Vgl. etwa Schamirs Knesset-Rede vom 10.8.1988. Dasselbe wurde auch im Wahlkampf programm des Likud deutlich. Schamir übertraf in dieser Orientierung sogar seinen Vorgänger Begin. Er stimmte gegen das von Begin ausgehandelte Vertragswerk von Camp David, weil darin die Abtretung (Rückgabe) des Sinai an Ägypten vorgesehen war.

43 Vgl. AdG, 3.1.1985, S. 28349. 
fehlenden Verläßlichkeit intemationaler Garantien. ${ }^{44}$ Auch sechs Jahre später gab es Hinweise, daß gerade die militärischen Anfangserfolge des ägyptischen Heeres akute Ängste vor "einer sich nähernden Schoah" (hebr. für Holocaust) wachriefen, 45 auch wenn der Autor der erwähnten Untersuchung dies für die 1973 verantwortlichen Führungspersönlichkeiten so nicht mehr feststellen zu können meinte. 46 Selbst das allmähliche Verschwinden der Generation der unmittelbar Betroffenen hat bis heute bemerkenswerterweise zu keiner Verringerung des entsprechenden Bewußtseins geführt. Mehr noch, neuere Umfragen unter israelischen Schülern ergaben, daß die Sprößlinge orientalischer Familien ihren europäisch-stämmigen Klassenkameraden darin nicht nachstehen, obwohl ihre Familien von der Vemichtung ja nicht betroffen waren. 47 Die Holocaust-Problematik und ihre Ubertragung auf den Konflikt mit den arabischen Staaten waren und sind fest im israelischen Kollektivbewußtsein verankert48 - und damit auch in der Politik Israels. Die Zerstörung des irakischen Atomreaktors Osirak durch einen Luftangriff im Juni 1981 z.B. wurde von dem damaligen Ministerpräsidenten Begin mit der Abwehr eines neuen Holocausts bzw. einer existentiellen Bedrohung der israelischen Nation begründet. 49

Sicherlich gab es wenige Führungspersönlichkeiten in Israels Geschichte, deren Denken so unmittelbar in den Kategorien von Holocaust und Weltkriegszeit ablief wie das Menahem Begins. 50 Aber wenn seine Nachfolger und speziell die Politiker der AP im Zusammenhang mit möglichen Bedrohungen Israels in den vergangenen Jahren nicht mehr so oft unmittelbar den Holocaust beschworen, so mag dies auch auf die realtiv entspannte Sicherheitslage zurückzuführen sein. Die Bindung destruktiver arabischer Energien durch den Golfkrieg ist ja von israelischen Politikem mit berechtigter Erleichterung betrachtet worden.

Aus dieser Entspannung darf aber keinesfalls der Schluß gezogen werden, daß das "Holocaust-Syndrom", die Perzeption einer existentiellen Bedrohung von Staat und Bürgem, überwunden worden sei. Deutliche Hinweise darauf liefern die stets heftigen Reaktionen der Politiker und des von ihnen geführten Staatsapparates auf Angriffe palästinensischer Freischärler und auf terroristische Überfälle im In- und Ausland. Die Sensibilität ist hier über alle Parteigrenzen hinweg anhaltend sehr hoch, obwohl solche Angriffe im Vergleich

44 Vgl. Brecher, Michael, Decisions in Crisis. Israel 1967 und 1973, Berkeley u.a. 1980, S. 344ff.; Seligmann, Israels Sicherheitspolitik, s. 178.

45 Vgl. Ben-Porat, Yehoschua u.a., Hamechdal (das Versäumnis), Tel Aviv 1974, zit. nach Neustadt, Israels zweite Generation, S. 33.

$46 \mathrm{Vgl}$. Brecher, Decisions. Alle anderen Elemente einschließlich eines anfänglichen starken Einbruches im Selbstvertrauen der Beteiligten wurden jedoch wiederum nachgewiesen.

47 Vgl. Neustadt, Israels zweite Generation, S. 28, Anm. 16 und S. 144.

48 ebd., S. 35.

49 Vgl. AdG 1981, S. 24639; Perlmutter, The Life and Times, S. 362.

50 Vgl. ebd.: Schiff, Ze' ev/Ya'ari, Ehud, Israel's Lebanon War, New York 1984, S.39. 
zum Ansturm der arabischen Armeen in früheren Kriegen doch unbedeutend wirken und sich der Konflikt mit den arabischen Staaten weitgehend auf die Bereiche Dioplomatie und Handel verlagert hat. Nur vor dem Hintergrund dieser Empfindlichkeit läßt sich daher erklären, daß unverhältnismäßige Rachemaßnahmen nach Terroranschlägen, wie die Bombardierung von Dörfern und palästinensischen Flüchtlingslagem und ganze Feldzüge, von der breiten israelischen Offentlichkeit stets mitgetragen werden. In demselben Zusammenhang ist auch die vielfach lautgewordene Kritik an der "Politk der eisemen Faust" von Verteidigungsminister Rabin zu verstehen. Intemational wegen ihrer Brutalität gegen die unbewaffnete Bevölkerung gebrandmarkt, galt sie besonders den Anhängem von Likud und den übrigen Rechtsparteien in Israel noch als zu weich.51

Die Beendigung des Golfkrieges und die irakischen Giftgas-Angriffe gegen iranische Truppen, kurdische Freischärler und Zivilbevölkerung haben ihren Betrag dazu geleistet, daß die Perzeption einer existentiellen Bedrohung in Israel noch in absehbarer Zukunft wirksam bleiben wird. 52

\section{Zum israelischen Dogma der Wehrhaftigkeit des Staates}

In der israelischen Gesellschaft hat sich unter dem Eindruck realer und subjektiv empfundener Bedrohung eine gewisse Festungsmentalität herausgebildet. Diese hat wohl drei wesentliche Bestandteile von beträchtlicher politischer Relevanz.

Das ist erstens die Wahmehmung, sich keinesfalls auf irgendwelche politischen Garantien verlassen zu können, sondern die eigene Sicherheit im Zweifelsfall allein gewährleisten zu müssen. Daraus folgt zweitens die feste Ưberzeugung, daß "Israel unbedingt sehr stark" und mehr noch: all seinen Gegnem überlegen sein müsse, wenn es überleben wolle. Nicht zuletzt als Reflex auf die vielen Israelis geradezu unverständliche "Opferlamm-Mentalität" der europäischen Juden gegenüber ihren Verfolgem wurden drittens Aktivität und Initiative schlechthin zu Dogmen. Im Licht der geschilderten Dogmen ist es gerade in der Auseinandersetzung mit den Arabem Israels Regierenden auferlegt, "jegliche Formen der Hilflosigkeit und Resignation zu bekämpfen" und sofort eine angemessene Antwort auf jede drohende Gefahr parat zu haben.53 Dadurch erklären sich spektakuläre Aktionen wir die Geiselbefreiung von Entebbe, die Zerstörung des irakischen Reaktors oder die Kommando-

51 Vgl. NZZ, 16.4.1988, S. 4; Jerusalem Post, 25.10.1988, S. 2.

52 Vgl. LM, 11.8.1988, S. 5.

53 Vgl. Neustadt, Israels zweite Generation, S. 138. 
überfälle auf die PLO-Hauptquartiere in Beirut und Tunis samt der dazu abgegebenen markigen Erklärungen der Verantwortlichen.54

Schicksalsergebenheit hat im modernen Israel ihren Platz unter den alten jüdischen Kardinaltugenden an andere verloren. So basieren israelische Erziehungsleitsätze heute offensichtlich auf dem Prinzip des "immer Kämpfen-, Gewinnen- und Siegen-Müssens".55 Das paßt in der Tat besser zu den Themen "Sicherheit, Verteidigung und Heldentum", die in Anlehnung an Shmuel Eisenstadt wohl als Kernelemente der neuen "jüdischen Kollektividentität" bezeichnet werden können. Das Symbol dafür ist vor allem die israelische Verteidigungsarmee.56 Die 1982/83 im Zusammenhang mit dem Libanon-Krieg laut gewordene Kritik an der Armee und besonders Verteidigungsminister Ariel Scharon ist weitgehend vergessen. Sie hat offensichtlich keine bleibenden Veränderungen bewirkt. 57 Wer auch nur den Anschein erweckt, nicht das Äußerste zur Erhaltung der Sicherheit getan zu haben, bekam und bekommt sehr schnell den israelischen Volkszorn zu spüren. Die Proteste gegen den hochverdienten General a.D. und Verteidigungsminister Mosche Dajan nach dem Krieg von 1973 oder auch gegen Verteidigungsminister Rabin wegen der fortdauernden Intifada sind hierfür typisch.58

Ungeachtet tagespolitischer Entwicklungen kann also für Israel ein grundlegender Konsens über die hohe Priorität von Sicherheit festgestellt werden. Er beruht auf einer allgemein verbreiteten Perzeption fortdauernder und existentieller Bedrohung. Vom Staat wird erwartet, daß er dieser Bedrohung mit allen Mitteln und rücksichtslos entgegenzutreten hat.

\section{Fazit}

Die Ursache dafür, daß beide große Parteien in Israel noch immer eine Friedenslösung ohne Beteiligung der PLO und ohne einen souveränen Palästinenserstaat anstreben, hat ihre Ursache nicht nur in tiefverwurzelten Bedrohungsperzeptionen, sondern auch in der Überzeugung von einer höheren Legitimität nationalstaatlicher Ambitionen der Juden und in religiösen und kulturellen Bedürfnissen.

54 Vgl. z.B. die einander sehr ähnlichen Erklärungen von Ministerpräsidentin Golda Meir im April 1973 (Jerusalem Post, 11.4.1973, S. 2) und Verteidigungsminister Rabin im Oktober 1985 (SWB/ME, 3.10.1985).

55 Vgl. Fleischhauer, Inge/Klein, Hillel, Über die jüdische Identität, Königstein 1978, zit. nach: Neustadt, Israels zweite Generation, S. 105.

$56 \mathrm{Vgl}$. Eisenstadt, Die Transformation, S. 265.

$57 \mathrm{Vgl}$. SchifflYa' ari, Israel's Lebanon War, S. 305f. Scharons politische Karriere ist bezeichnenderweise von dem Krieg kaum beeinträchtigt worden.

58 Vgl. Meir, Mein Leben, S. 288; Jerusalem Post, 11.4.1988, S. 1. 
Die PLO wird in Israel als Verkörperung der unerbittlichen Feindschaft der arabischen Welt gegen Israel gesehen, ihre Ziele als den eigenen diametral entgegengesetzt bewertet.

Wenn sich heute eine zunehmende Zahl von Israelis für Verhandlungen mit der PLO ausspricht, so ist das wohl vor allem eine Reaktion auf die untragbaren Verhältnisse in den besetzten Gebieten. Sie berufen sich dabei nämlich insbesondere auf die wirtschaftlichen Folgen und die Gefahren für die Identität des jüdischen Staates. In ihrer Einschätzung der PLO bleiben sie jedoch skeptisch, so daß von einer grundlegenden Änderung ihrer Perzeption der Palästinenserorganisation wohl keine Rede sein kann.

Vor diesem Hintergrund wird deutlich, da $\beta$ die israelischen Entscheidungsträger bei der Formulierung ihrer Palästina-Politik lebenswichtige Interessen, aber auch die psychologischen und kulturellen Bedürfnisse einer hochsensibilisierten Wählerschaft berücksichtigen müssen, soweit sie sie nicht ohnehin teilen. Dazu kommen komplizierte innenpolitische Machtverhältnisse. All dies macht es den israelischen Regierungsparteien und insbesondere dem Likud und der Arbeiterpartei schwer, jene Kursänderung vorzunehmen, die heute im Interesse einer Annäherung an eine Friedenslösung im Nahen Osten erforderlich zu sein scheint. 


\section{Israel's Attitude towards the Palestinian Question - Concept and Background}

\section{By Peter Gottstein}

The Palestinian uprising triggered important changes among which PLO recognition of Israel's right to exist and the subsequent initiation of a dialogue between the PLO and the United States rank supreme. The PLO has mustered wide support for its political efforts. Israel has refused to revise its Palestina Policy thus disappointing some governments traditionally friendly to her. Despite this change of position neither the Labour nor the Likud, in power since 1954, have provided an opening for a compromise with basic demands of the PLO. Both Palestinians and Israelis claim a holy right to the possession of Eretz Israel and Palestine respectively. The PLO challenges the raison d'être of the Jewish State, while the Jewish State would like to ensure the physical security of the Jewish people against the possibility of another Holocaust. To many the PLO Arab behaviour has been judged as anti-semitic. Their hostile actions against the State of Israel were seen as preparations for a new Holocaust. If today a growing number of Israelis advocates negotiations with the PLO, this is probably motivated less by changing perception of the PLO than by the intolerable situation in the occupied territories. 